THERAPEUTICS

\section{Split personality}

Tamoxifen - a selective oestrogen receptor modulator (SERM) that regulates oestrogen receptor (ER)-induced gene expression - has a split personality: it inhibits gene expression, and hence cell growth, in the breast, but promotes it in the endometrium. Treating breast cancer patients with tamoxifen might therefore promote carcinogenesis in the uterus. So what determines these tissue-specific effects? Yongfeng Shang et al., reporting in Science, show that tissue-specific co-regulator expression levels determine tamoxifen's effects.

The ER regulates gene transcription both directly, by binding to an oestrogenresponsive element (ERE) in gene promoters, and indirectly, by binding through other transcription factors. How do SERMs modulate these different promoter types? Tamoxifen and raloxifene - a SERM that acts as an oestrogen antagonist in both breast and endometrium - were administered to MCF-7 breast carcinoma cells and Ishikawa endometrial carcinoma cells, to investigate their effect on gene expression. Tamoxifen, but not raloxifene, stimulated transcription of the indirect ER target genes c-MYC and $I G F 1$, but not the direct ER target genes CTSD and EBAG7, in the Ishikawa cells, but not in the MCF-7 cells. The promoter type is therefore an important determinant of tamoxifen activity.

But what mediates this differential activity? Chromatin immunoprecipitation experiments showed that tamoxifen recruited transcriptional repressors to both promoter types in MCF-7 cells, and to the direct promoters in Ishikawa cells. However, tamoxifen recruited transcriptional activators to indirect promoters in Ishikawa cells. Might different expression levels of these transcriptional regulators explain the

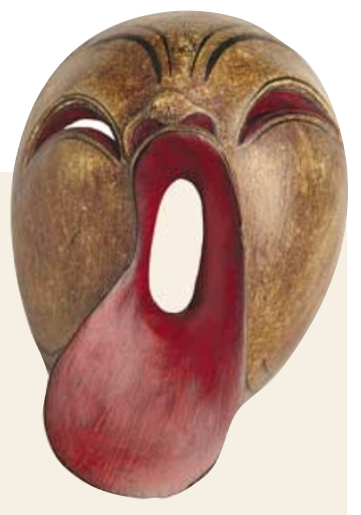

tissue-specific effects of tamoxifen? Levels of the transcriptional activator NCOA1 (also known as SRC1) were lower in mammary cells than in endometrial cells. Overexpression of NCOA1 in MCF-7 cells stimulated tamoxifen-induced transcription. By contrast, its silencing in Ishikawa cells by RNA interference abolished tamoxifen-induced transcription and the associated cell-cycle progression.

So, tissue-specific differences in NCOA1 levels determine whether tamoxifen is pro- or anti-oestrogenic on some promoter types. Let's hope that this mechanistic knowledge will lead to better SERMs without carcinogenic side effects. Emma Greenwood

(2) References and links ORIGINAL RESEARCH PAPER Shang, Y. \& Brown, M. Molecular determinants for the tissue specificity of SERMs. Science 295, 2465-2468 (2002)

\title{
EPIDEMIOLOGY
}

\section{No-win situation}

Human papillomavirus (HPV) is a key cause of cervical cancer, but is not seen as being the only factor, as cervical infections with HPV are very common and usually only transient. In cases that

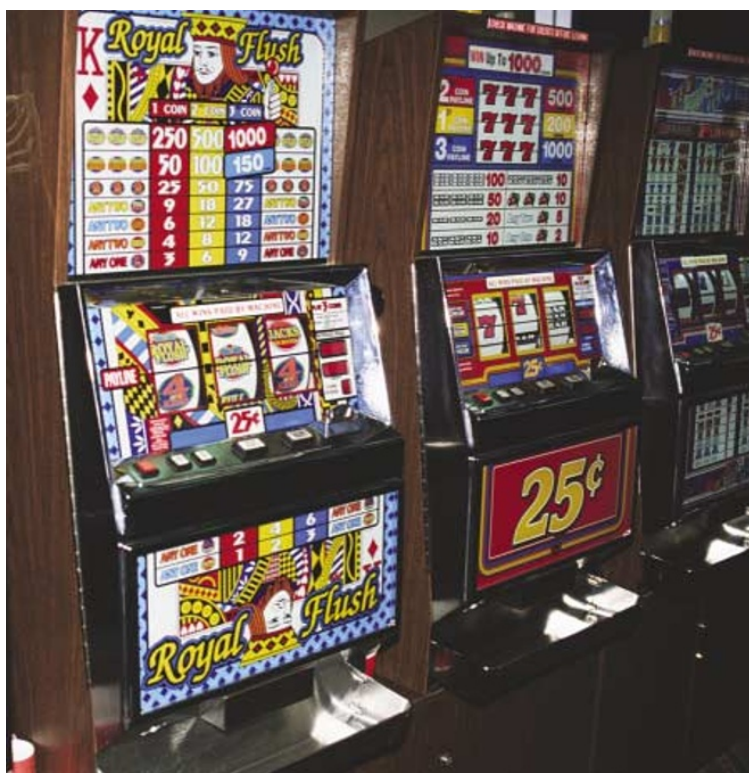

persist and progress to cervical intraepithelial neoplasia (CIN) and invasive cancer, there must be some other factor(s) involved that create a predisposition. The International Agency for Research on Cancer (IARC) Multicentric Cervical Cancer Study Group now confirm that two veteran suspects - the use of oral contraceptives and high parity — are independent risk factors for cervical cancer.

Oral contraceptives and high parity have long been suspected risk factors for cervical cancer, but most previous studies have been confounded by other variables, such as sexual behaviour. In addition, although HPV is almost always present in cervical cancer, previous studies have not taken HPV status into account, thereby potentially biasing the results.

The authors used a pooled analyis of case-control studies of in situ carcinoma and invasive cervical cancer in individuals from four continents, and controls who were HPV positive. Polymerase chain reaction amplification, targeting the HPV L1 gene, was used to detect and type HPV DNA in cells exfoliated from the cervix or from biopsy specimens.

In women who used oral contraceptives, the risk of developing cervical cancer increased with duration of use, from no increased risk at less than 5 years to a fourfold increase after more than 10 years, compared with women who had never used oral contraceptives. In the second study, a 2.3 -fold risk increase was seen in women after seven pregnancies compared with those who had only had one or two; and a 3.8 -fold increase was seen in women after seven pregnancies compared with those who had never been pregnant.

There is clearly an association between oral contraceptive use/parity and cervical cancer risk. The IARC Study Group also showed that there was no association between either oral contraceptive use or parity and the detection of HPV positivity among the individuals that were being selected as potential controls, indicating that oral contraceptives and parity act, not by increasing the acquisition or persistence of HPV infection, but by independently promoting the progression of infection to CIN and invasive cancer. How this works has yet to be elucidated, but the answers are very much needed, especially in the developing world where cervical cancer is widespread and reliable cytological screening is sparse.

Sandra Clark

\section{(Q) References and links}

ORIGINAL RESEARCH PAPERS Moreno, V. et al. Effect of ora contraceptives on risk of cervical cancer in women with human papillomavirus infection: the IARC multicentric case-control study. Lancet 359, 1085-1192 (2002) | Munoz, N. et al. Role of parity and human papillomavirus in cervical cancer: the IARC multicentric case-control study. Lancet 359, 1093-1101 (2002) 